\title{
FOUR DEGREE OF FREEDOM MEASURING INSTRUMENT FOR PRECISION MACHINERY BASED ON SEMICONDUCTOR LASER OPTICAL FIBER COMPONENTS
}

\author{
Shuiquan $\mathrm{ZHU}$ \\ Tianjin Electronic Information college, Tianjin, China \\ Email: zhushuiquantianjin@163.com
}

\begin{abstract}
Based on the forthcoming six degree of freedom simultaneous measurement and research, a basic platform is set up, and a four degree of freedom simultaneous measuring instrument is developed. The instrument uses a semiconductor laser single mode fiber component as the light source, which effectively reduces the light drift of the laser itself. A high precision and high stability measurement reference ray is provided for the measurement of straightness and angle. Using the pyramid prism as a sensitive unit and reflecting beam backward, the structure volume of the instrument is reduced. The four-quadrant detector and position sensitive detector are used as photoelectric detection devices. The four-degree of freedom (straightness of $\mathrm{X}$ and $\mathrm{Y}$ direction, deflection angle and pitch angle) of the machine tool guide is measured at the same time by collecting and processing data through single chip microcomputer (SCM).
\end{abstract}

Keywords: Four degree of freedom; Semiconductor laser; Four-quadrant detector

\section{Introduction}

The precision detection of computer numeric control (CNC) machine tool and geometric position in machining center is an important means to ensure the machining precision and product quality. For a machine tool processing center with threedimension, there are 21 kinds of errors to be detected. These errors are: The 6-dimension error components under each axis are add to the perpendicularity error between the 3 axes. The error of the six degrees of freedom is: position error in the direction of three axes $(\Delta x, \Delta y, \Delta z)$ and angle error (pitching angle $\alpha$, deflection angle $\beta$, roll angle $\gamma$ ) around three coordinate axes. The error of these six degrees of freedom directly affects the quality of the machine tool parts. At present, dual frequency laser interferometer is mainly used in industrial testing.

Although the precision is high, only one error can be measured at a time. Moreover, the different accessories should be replaced each time, which brings a lot of inconvenience to the measurement (Loschaket al., 2016).

High tech industries such as aviation, aerospace, computer and integrated circuits all need a lot of precision and ultra-precision parts.

The manufacture of these parts must have high precision machine tools. In order to improve the precision of machine tools and meet the requirements of the development of precision machine tool processing industry, the method of on- line compensation (measuring the error of machine tool system by real-time online) need to be corrected at present. This requires a measuring instrument with convenient, reliable, high precision and low cost.

\section{Development of a Four Degree of Freedom Measuring Instrument}

Laser 4D measuring instrument is suitable for geometric precision measurement of large precision equipment (CNC machine tool and coordinate measuring machine). The error parameters on the four degrees of freedom of the linear axis can be measured at a time by one installation, including horizontal straightness (XX), vertical straightness (YY), pitching angle (Pitch) and deflection angle (Yaw).

The overall design of the instrument is shown in figure 1 . The system consists mainly of a probe (fixed part) and a target mirror (active part). The probe integrates optical system, 4D sensor unit, laser driver and signal processing circuit. The measurement datum is a beam emitted by a laser parallel to the surface of the device under test (DUT).

In the process of measurement, the target mirror is moved along the measured surface, and the laser beam emitted the laser is divided into two beams through the target mirror. A beam of light is reflected back through a pyramid reflex prism, which is used as a reference beam for straightness 
measurement. The relative displacement between the sensor and the reference beam is measured by a four-quadrant photoelectric receiver installed in the sensor.

The other beam is reflected by a semitransparent mirror, which is used as a reference beam for measuring angle error. It is received by a position sensitive detector in the sensor and used to measure angle error (pitching angle and pendulum angle).
The signal measured on the detector is processed by the analog digital conversion after the circuit process. The signal measured on the detector is processed by the analog digital conversion after the circuit is processed. It is sent to the computer by the SCM through the serial port and is displayed.

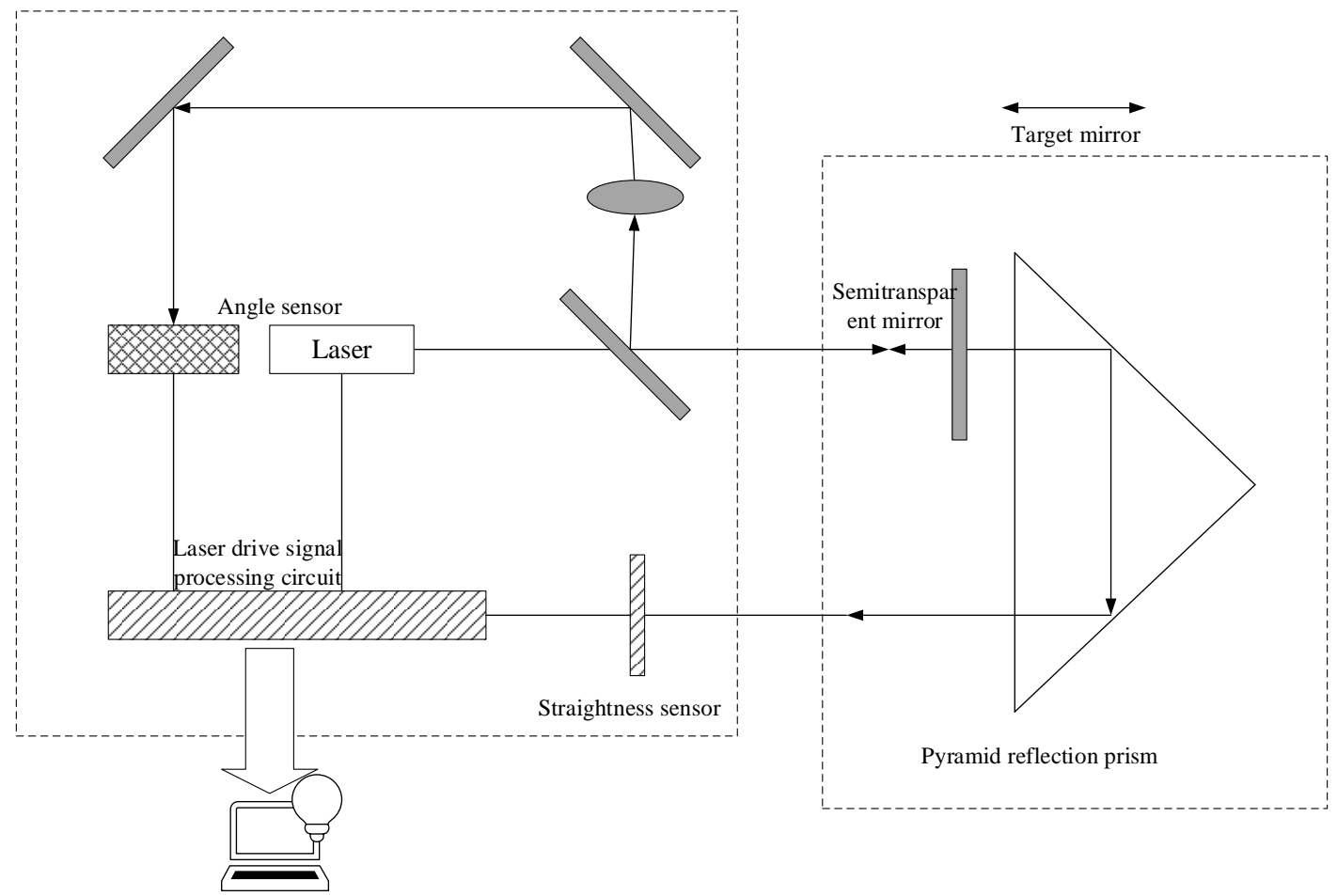

Figure 1. The overall design of the instrument

\section{The Measuring Principle of the Instrument}

Collimation measurement technology is used in the measurement system (Zudilovaet al., 2016). The collimation of semiconductor laser single mode fiber module is adopted. This technology is mature, widely applied and has high reliability. In addition, in recent years, the development of photoelectric detectors is becoming more and more mature. The control of microcomputer, data acquisition and processing have promoted the development and application of this traditional measurement method.

\subsection{Principle of straightness measurement}

As shown in figure 2, the measurement datum of the straightness measurement system (Selmiet al., 2014; Soriano et al., 2013) is that the laser sends out the beam parallel to the surface of the measured piece. When measuring, the pyramid prism moves along the measured surface, and the straightness error of the measured surface causes the relative movement between the pyramid prism and the laser beam.

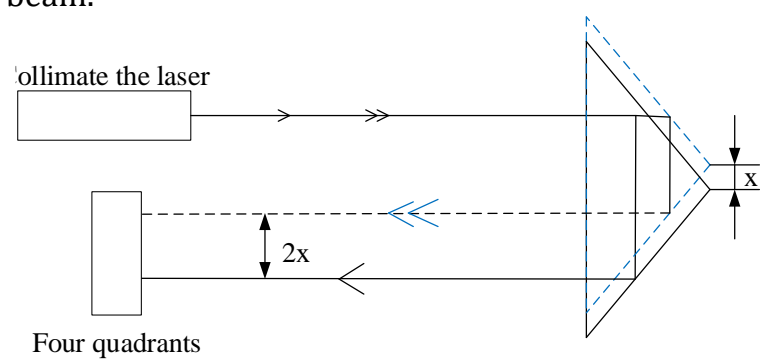

Figure 2. Principle of straightness measurement

As shown in figure 2, the measurement datum of the straightness measurement system (Selmiet al., 2014; Soriano et al., 2013) is that the laser sends out the beam parallel to the surface of the measured piece. When measuring, the pyramid prism moves along the measured surface, and the straightness error of the measured surface causes the relative movement between the pyramid prism and the laser beam. Thus, the position of the backward reflected beam to the receiving device (four-quadrant photo detector) has changed. 
When the pyramid prism has a $\mathrm{x}$ displacement, the spot center on the four-quadrant will move $2 x$. Through the signal processing circuit, the voltage signal corresponding to the straightness error can be obtained, and the straightness error can be measured after calibration

\subsection{Principle of angle measurement}

The angle measuring mechanism (Toomeyet al., 2014) is shown in figure 3.

The measured reference beam is reflected to probe by a semitransparent mirror.

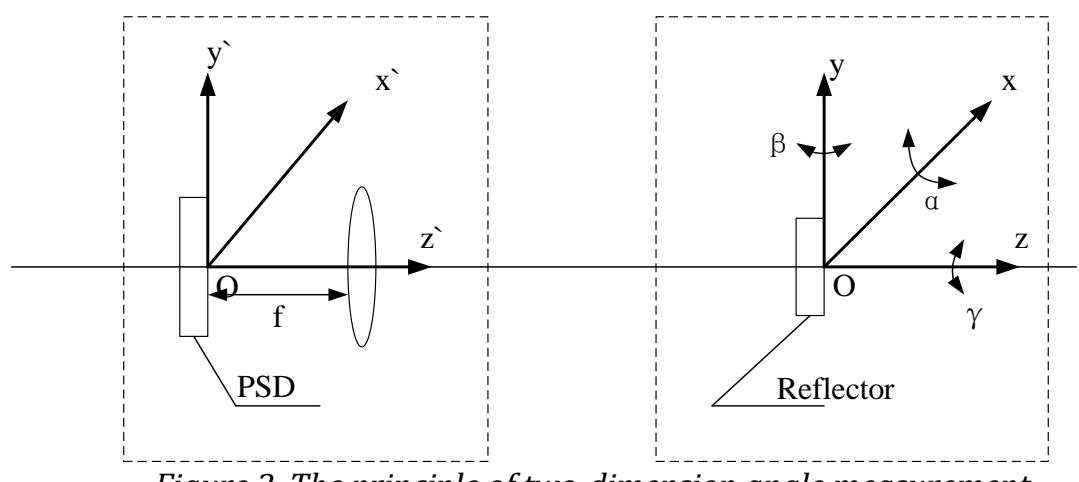

Figure 3. The principle of two-dimension angle measurement

The reflected light is imaged by the lens and is received by a position sensitive detector in the angle sensor. Any angle change will result in the shift of the image point on the detector. The pitch angle $(\alpha)$ and the pendulum angle $(\beta)$ can be obtained according to the formula (1):

$$
\left.\begin{array}{l}
\alpha=\Delta x /(2 f) \\
\beta=\Delta y /(2 f)
\end{array}\right\}
$$

In the formula, $\Delta x$ and $\Delta y$ are the offset of the image point in the horizontal and vertical direction, respectively. fis lens focal length.

When the target mirror is moving along a linear guide rail, the three-dimensional translation and rolling have no effect on the normal direction of the mirror. That is to say, the displacement $\triangle \mathrm{x}$ and $\triangle \mathrm{y}$ of the reflected light point on the detector PSD is insensitive to the translation of the three directions and the roll angle $\gamma$. It is sensitive only to the pitching angle $\alpha$ and the pendulum angle $\beta$, and the error separation is basically realized.

\section{The Composition of the Measurement System}

The measurement system is mainly composed of optical path, photoelectric detection device, circuit part and software part.

\subsection{Optical paths}

Because the divergence angle (below $1 \mathrm{mrad}$ ) of the output beam emitted the helium neon laser is small, the light source is close to the diffraction limit.

It has good mono-chromaticity (bandwidth is less than $20 \mathrm{~Hz}$ ) and coherence. Because the output light is visible light, the helium neon laser is usually used in laser alignment. However, He Ne laser has a large volume, high driving voltage, and complex maintenance and regulation. In particular, the size of the helium neon laser is large and does not meet the design requirements of miniaturization of this instrument.

With the development of semiconductor laser manufacturing technology (Tsiminis et al., 2013; Brunner et al., 2015), the conductor laser has gradually won the favor of the people with its superior performance.

Characteristics of semiconductor lasers: small structure, simple and sturdy, efficient, wide laser wavelength, long service life. It can be powered by a DC regulated power supply or battery, which simplifies the power supply design of laser.

However, the divergence angle of the beam emitted by a semiconductor laser is large. The ejection beam is an elliptical beam, which is not conducive to the collimation measurement.

Therefore, a semiconductor laser optical fiber component is used in the design process. The semiconductor laser fiber component is composed of a semiconductor laser and a single mode fiber.

The flat drift and angular drift of the output beam emitted by the laser only affect the coupling efficiency and do not affect the direction of the output beam.

Meanwhile, the laser beam is affected by single mode fiber transmission and collimating lens, the elliptical beam is shaped into a collimated beam with circular cross-section, which provides a stable baseline for measurement.

In addition, using a semiconductor laser optical fiber component, the volume of the system is reduced and the stability and collimation accuracy of the system are increased. The FMXL635-001SF0 type laser provided by Blue sky Company of the United States is used in this system, its main photoelectric parameters are shown in table 1 : 
Table 1. Main parameters of the laser

\begin{tabular}{|c|c|c|c|c|c|c|}
\hline Project & Symbol & Unit & $\begin{array}{c}\text { Minimum } \\
\text { value }\end{array}$ & $\begin{array}{c}\text { Typical } \\
\text { value }\end{array}$ & $\begin{array}{c}\text { Maximum } \\
\text { value }\end{array}$ & Test condition \\
\hline $\begin{array}{c}\text { Ejection light } \\
\text { power }\end{array}$ & Pо & $\mathrm{mW}$ & 1.5 & 1.8 & & $\mathrm{Tc}=25^{\circ} \mathrm{C}$ \\
\hline $\begin{array}{c}\text { Threshold } \\
\text { current }\end{array}$ & Iтн & $\mathrm{mA}$ & & 25 & & $\mathrm{Tc}=25^{\circ} \mathrm{C}$ \\
\hline $\begin{array}{c}\text { Working } \\
\text { current }\end{array}$ & $\mathrm{I}$ Iо & $\mathrm{mA}$ & & 30 & & $\mathrm{Tc}=25^{\circ} \mathrm{C}$ \\
\hline $\begin{array}{c}\text { Working } \\
\text { voltage }\end{array}$ & $\mathrm{V}_{\mathrm{OP}}$ & $\mathrm{V}$ & & & 2.7 & $\mathrm{Tc}=25^{\circ} \mathrm{C}$ \\
\hline $\begin{array}{c}\text { Output } \\
\text { wavelength }\end{array}$ & $\gamma$ & $\mathrm{Nm}$ & 635 & 635 & 640 & $\mathrm{Tc}=25^{\circ} \mathrm{C}$ \\
\hline $\begin{array}{c}\text { Monitoring } \\
\text { current }\end{array}$ & $\mathrm{IMON}$ & $\mathrm{mA}$ & & 0.15 & & $\mathrm{Tc}=25^{\circ} \mathrm{C}$ \\
\hline
\end{tabular}

Figure 4 is its object and its internal structure.

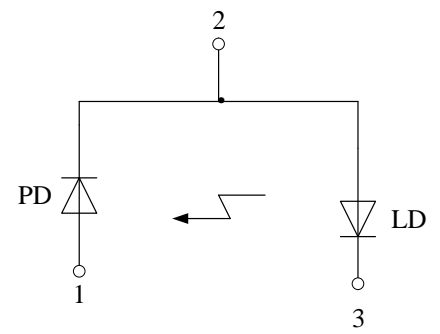

Figure 4. Internal structure diagram of laser

The laser has three pins, including pin 1, pin 2 and pin 3. Laser diode (LD) is located between pin 2 and pin 3 as a luminescent light source. Photo detector (PD) is located between pin 1 and pin 2and used for feedback control of output light power.

When the laser power is increased, the reverse current of the PD is increased to reduce the current passing through the laser. Otherwise, when the output power of the laser is reduced, the reverse current of PD decreases.

As a result, the current passing through the laser increases, forming a negative feedback. It has achieved the effect of stabilizing the output light power emitted by laser.

\subsection{Optoelectronic detection device}

With the development of optoelectronic technology, a large number of new optoelectronic devices have been widely used. These devices can not only detect the conventional radiation light power, but also have the ability to distinguish the space, which can be used to detect the location of the incident points, the spatial and dark distribution.

In the field of measurement, charge coupled device (CCD), four quadrant detector and position sensitive detector (PSD) are commonly used. In this system, the four-quadrant detector is used in straightness measurement and position sensitive detector is used in angle measurement. In essence, the four-quadrant detector is a junction type optoelectronic device with large area.

As shown in figure 5, it uses photolithography to divide a circular photosensitive surface into 4 fanshaped areas. The 4 areas have a common electrode. Each region is equivalent to a photodiode. In the ideal case, each photodiode should have the same performance parameters. The size of the photocurrent in each region depends on the distribution function of the area and intensity of the light spot on it.

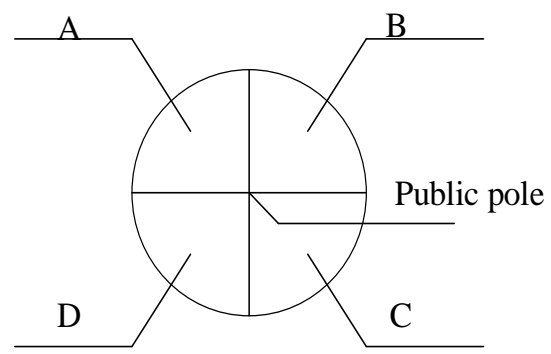

Figure 5. Four quadrant schematic diagram

When the spot diameter is larger than the width of the optical line and is smaller than the total area of the detector, the location of the spot can be deduced through the proportional relationship between the photocurrent in each area.

$$
\left.\begin{array}{l}
X=\frac{(A+D)-(B+C)}{A+B+C+D} \\
Y=\frac{(A+B)-(C+D)}{A+B+C+D}
\end{array}\right\}
$$

The four-quadrant detector used in our instrument is the SPOT-9DMI detector provided by UDT Company.

It has the advantages of wide working temperature and fast response speed, while the sensitivity of position response is better than $0.1 \mu \mathrm{m}$. 


\subsection{Circuit part}

Circuit design is one of the main tasks of the paper, including laser driving circuit design, photoelectric detector signal reception and processing circuit design, and computer interface circuit design.

Laser dive circuit: As the instrument is stable, the light intensity emmited by a semiconductor laser must be stable. For this purpose, as shown in figureb 6 , a laser drive circuit is designed.

When the light intensity of the laser LD increases, the current passing through the photodetector PD increases.
The base current of the triode $\mathrm{V} 1$ and the collector current of V1 increase. The collector voltage of V1 and the base current of the triode V2 decrease.

The collector current (the laser current) decreases at the same time, reaching the purpose of reducing the intensity of light. Otherwise, when the laser intensity is reduced, the intensity of light can be increased by the control of PD.

The driving circuit above has formed a closed loop control system, which achieves the purpose of stabilizing the laser intensity.

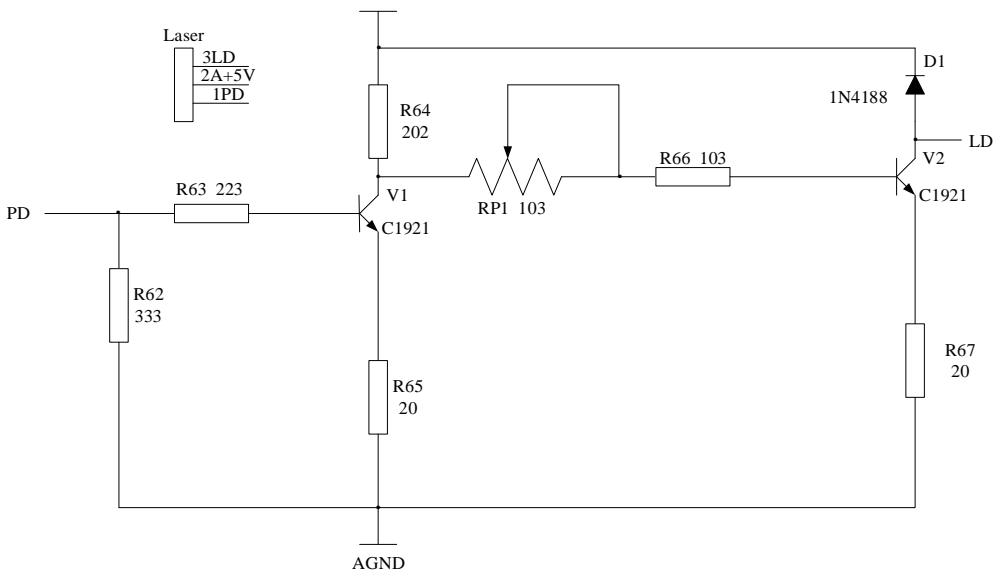

Figure 6. Laser drive circuit

Signal processing circuit of photodetector: The output of a photodetector is an analog current signal. After converting it into a digital signal, it is sent to the computer for processing.

The processing circuit of four quadrant detector: The common pole of the CATH detector provides the bias voltage. The A, B, C, and D are connected to the four quadrant output of the detector respectively.

The four circuit current signals of the detector are converted into four circuit voltage signals out5, out6, out7 and out8 by dual operational amplifier LF412, so as to facilitate AD conversion.

He signal processing circuit of position sensitive detector PSD: The pin 1 and pin 2 connect the PSD anode, while the pin 3 and the pin 4 connect the PSD cathode to provide the voltage offset, which is the same as the four quadrant.The current signal of PSD output is converted to voltage signal out1, out2, out3, out4 by dual operational amplifier LF412.

Analog digital conversionand SCM part: The two detector has a total of 8 signals for AD conversion.

The AD conversion chip used by this instrument is the MAX197 provided by MAXIM company of the United States. MAX197 has a standard microcomputer interface. The three state data I/O port is used as a 8 bit data bus, and the timing of the data bus is compatible with the general microprocessors. All logical inputs and outputs are compatible with the TTL/CMOS level. The main features are: Single $+5 \mathrm{~V}$ power supply, 12 bit resolution,error $\pm 1 / 2$ (LSB), 8 analog input channel.

The input range can be selected in different ranges by software $( \pm 10 \mathrm{~V}, \pm 5 \mathrm{~V}, 0 \mathrm{~V}-10 \mathrm{~V}, 0 \mathrm{~V}-5 \mathrm{~V})$. The input overvoltage tolerance of the converter is $\pm 16.5 \mathrm{~V}$. The conversion time is $6 \mu$ sand sampling rateis $100 \mathrm{kSPS}$.

The internal or external clock, internal or external acquisition, and internal $4.096 \mathrm{~V}$ voltage reference or external voltage reference can be selected by software.The low power operation mode can be selected through the SHDN pins and software.

The pins and wiring of MAX197 are shown in figure 7. The $\mathrm{CHO}-\mathrm{CH} 7$ is connected to the voltage signal of the 8 path detector as the input channel of the analog signal. D0-D7 is used as a 12 bit digital signal output channel, in which D0-D3. When HBEN=0, D0-D3 is output. When HBEN=1, the D8D11 is exported and the data bus is reused. D0-D7 is also a writing channel for the SCM control signal.

When the operation is started, the conversion begins. The write operation will select multiple channels. It is also necessary to determine whether the input range of MAX197 is monopole or bipolar. A write pulse $(\mathrm{WR}+\mathrm{CS})$ can start a collection, or 
initialize the sample and start the conversion. When the conversion is finished and a correct result is produced, the chip sends out a standard interrupt signal INT (as a recognition signal) to the SCM.INT becomes a high level in the first reading cycle or writing a new control byte.

The micro controller used in this instrument is the AT89C2051 single chip provided by ATMEL Company. AT89C2051 has 20 pins, 2 bidirectional $\mathrm{I} / 0$ interfaces $\mathrm{P} 1$ and $\mathrm{P} 3$ interface, $2 \mathrm{kB}$ Flash memory, 128 byte internal RAM, 5 interrupt sources, two 16 bit timer / counters, programmable serial interface and on-chip clock oscillator. The automatic reset mode is adopted, and the clock signal adopts the internal oscillation mode.

The P1 port acts as the input port of the AD chip data signal. P3.3, P3.4 and P3.7 are used for the output port of the AD chip working control signal. P3.2 is used to read the interrupt signal sent by the AD chip when AD conversion is completed. P3.0 and P3.1 communicate with the computer as serial port.

The workflow of SCM is shown in figure 7:

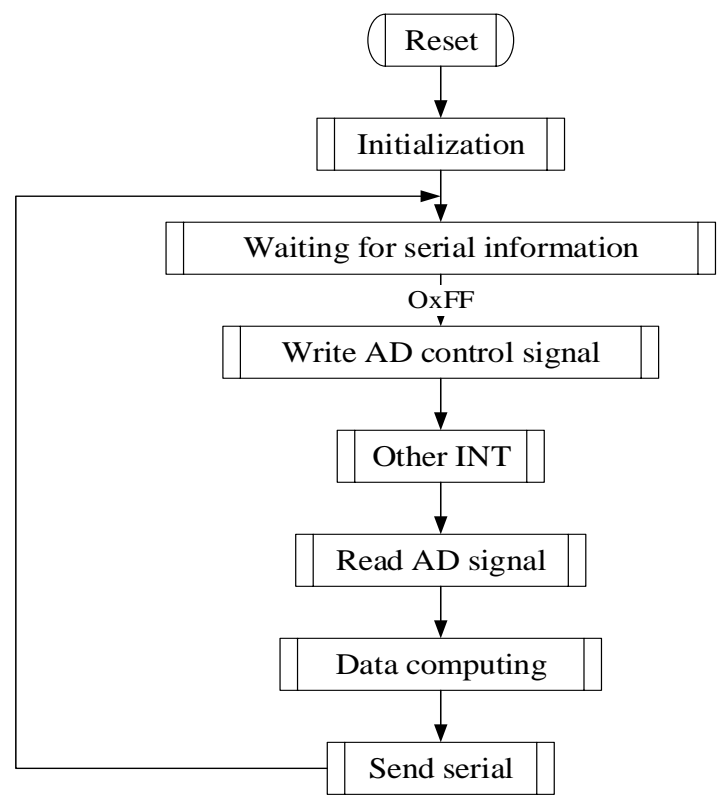

Figure 7. The workflow of SCM

After the SCM is reset, it initializes first, then sets the work mode of interruption, serial port and so on.After the initialization is completed, the serial port which is sent by the computer starts to collect the signal. When the serial message is $0 x F F$, the control signal is written to the AD chip.

When the SCM detects the interrupt signal INT produced by the $\mathrm{AD}$ conversion, the $\mathrm{AD}$ signal is read. The signals that are read are combined and calculated as voltage values, then the siginals are sent to the computer through the serial port to continue to wait for the starting signal of the computer.

\section{Software Part}

The software part of the computer is written by Visual C++. The A, B, C, and D of the PSD are respectively the voltage values of the four pins of the PSD. $\alpha$ and $\beta$ are the intermediate values of angle calculated by A, B, C, D. $\alpha(\mathrm{s})$ and $\beta(\mathrm{s})$ are measured values of actual angle errors calculated by $\alpha$ and $\beta$.

The QD part is the same. A, B, C, and D are respectively the voltage values of the four pins of the QD. X and $Y$ are the middle values of the straightness calculated by $A, B, C, D . X(\mu m)$ and $Y(\mu m)$ are measured values of the actual straightness error calculated by $\mathrm{X}$ and $\mathrm{Y}$ after calibration.

The software is operated on the serial port through the MSComm control, and the serial port is initialized first after the software is started. The software can be collected manually or automatically.

After the manual collection button is clicked, the computer sends a data acquisition signal to the SCM through the serial port. After the data of the SCM is obtained, the data is calculated and the intermediate value is obtained. Then, the final four degree of freedom error measurement data are obtained by checking the interpolation table. After refreshing the edit box, all kinds of data are displayed on the software interface. Manual acquisition can be used for fixed point measurement.

Automatic acquisition is to repeat the operation of manual acquisition, and can be used to measure the stability of the instrument.

\section{Conclusion}

In view of how to measure these errors quickly and accurately, the development of the four degree of freedom simultaneous measuring instrument is carried out. A system structure based on optical fiber component of semiconductor laser is designed, which can measure four degree of freedom simultaneous measuring instrument and the probe without cable connection.

The selection of optical components, the main circuit parts and the design of the software are also carried out.

\section{References}

[1] Loschak, P. M., Degirmenci, A., Tenzer, Y., et al., (2016). A Four Degree of Freedom Robot for Positioning Ultrasound Imaging Catheters. Journal of Mechanisms \& Robotics-transactions of the Asme, 8(5):0510161-510169.

[2] Zudilova, T. V., Ivanov, S. E. (2016). Mathematical modeling of the robot manipulator with four degrees of freedom. Global Journal of Pure \& Applied Mathematics, 12(5), 4419-4429. 
[3] Selmi, F, Braive, R,, Beaudoin, G, et al. (2014). Relative refractory period in an excitable semiconductor laser. Physical review letters, 112(18): 183902.

[4] Soriano M C, García-Ojalvo J, Mirasso C R, et al., (2013). Complex photonics: Dynamics and applications of delay-coupled semiconductors lasers. Reviews of Modern Physics, 85(1): 421.

[5] Toomey, J. P., Kane D. M. (2014). Mapping the dynamic complexity of a semiconductor laser with optical feedback using permutation entropy. Optics express, 22(2): 1713-1725.

[6] Tsiminis, G., Wang, Y., Kanibolotsky, A. L., et al., (2013). Nanoimprinted Organic Semiconductor Laser Pumped by a Light-Emitting Diode. Advanced Materials, 25(20): 2826-2830.

[7] Brunner, D., Soriano, M. C., Porte, X., et al., (2015). Experimental phase-space tomography of semiconductor laser dynamics. Physical review letters, 115(5): 053901.

\section{INCDMTM INNOVATIVE PRODUCTS:}

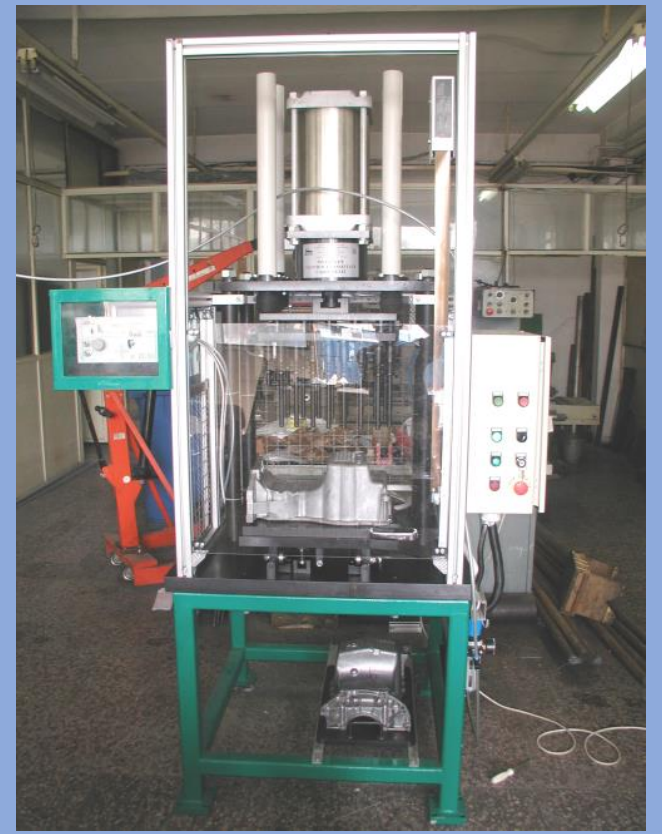

TIGHTNESS CONTROL EQUIPMIENT FOR OIL PAN 